\title{
Relationships among Some Types of Fuzzy Soft Open Sets in Fuzzy Soft Tri-Topological Spaces
}

\author{
Asmhan F. Hassan \\ Department of Mathematics, \\ Faculty of Education for Girls, \\ University of Kufa, Iraq
}

\author{
Azhaar A. Farhan \\ Department of Mathematics, \\ Faculty of Education for Girls, \\ University of Kufa, Iraq
}

\begin{abstract}
In the present paper, we continue the study on fuzzy soft tritopological spaces, and investigate the relationships among some main types of fuzzy soft open sets fuzzy soft tritopological spaces. In particular, we study the relationships among the Fuzzy soft $\tau_{1} \tau_{2} \tau_{3}$-open (closed) set, Fuzzy Soft Tri- $\alpha$-open (closed) set, Fuzzy Soft Tri-pre-open(closed) set and Fuzzy Soft Tri-semi-open (closed) set in Fuzzy Soft Tritopological Spaces. And We analyze the relationships among these notions by providing theorems and counter examples.
\end{abstract}

\section{General Terms}

2010 AMS Classification: 54A05, 54A10, 54E55, 54E99.

\section{Keywords}

Fuzzy Soft Tri-topological space, Fuzzy soft $\tau_{1} \tau_{2} \tau_{3}$-open (closed) set, Fuzzy Soft Tri- $\alpha$-open (closed) set, Fuzzy Soft Tri-pre-open(closed) set, Fuzzy Soft Tri-semi-open (closed) set.

\section{INTRODUCTION}

In 1965, Fuzzy set was introduced by Zadeh in [1] as a mathematical way to represent and deal with vagueness in everyday life. And the applications of Fuzzy set theory can be found in many branches of sciences (see [2,3])

In 1999, Soft set theory was initiated by Molodtsov [4], he defined the concept of Soft set theory as a new mathematical tool, and presented several fundamental results and successfully applied it to several mathematical directions such as smoothness of functions, theory of probability, Riemannintegration, operations research, Perron integration, etc. A Soft set is a collection of approximate descriptions of an object. He also showed how Soft set theory is free from the parametrization inadequacy syndrome of Fuzzy set theory, rough set theory, probability theory and game theory. Some important applications of Soft sets are in decision making problems and information systems [5][6].

In 2001, Maji et al. [7] presented the concept of the Fuzzy Soft sets by embedding the ideas of Fuzzy sets. By using this definition of Fuzzy Soft sets many interesting applications of Soft set theory have been expanded by researchers. Roy and Maji [8] gave some applications of Fuzzy Soft sets. Aktas and Cagman [9] compared Soft sets with the related concepts of Fuzzy sets and rough sets. Yang et al. [10] defined the operations on Fuzzy Soft sets which are based on three Fuzzy logic operators: negation, triangular norm and triangular conorm. Xiao et al. [11] presented the combination of interval-valued Fuzzy set and Soft set.

In 1963, Kelly first initiated the concept of Bitopological spaces [12], where defined a Bitopological space to set with two topologies and initiated the systematic study of Bitopological spaces. In later, many researchers studied Bitopological spaces (see $[13,14]$ ) where carrying out a wide scope for the generalization of topological results in Bitopological environment.

In 2014, Ittanagi [15] introduced the concept of Soft Bitopological spaces, which is defined it over an initial universal set with fixed set of parameters, and he introduced some types of Soft separation axioms in Soft Bitopological spaces. A study of Fuzzy Soft Bitopological spaces is a generalization of the study of Fuzzy Soft topological spaces. In 2015, Mukherjee and Park [16] were first introduced the notion of Fuzzy Soft Bitopological space and studied some of their basic properties, and to more information (see [17,18]). In 2000, Kovar.[19], initiated the concept of Tri-topological spaces by modify $\theta$ - regularity for spaces with three topologies, where they define it as a spaces equipped with three topologies, i.e. triple of topologies on the same set, Palaniammal [20] studied Tri-topological spaces and introduced semi-open and pre-open sets in Tri-topological spaces and he also introduced Fuzzy Tri-topological space. In 2004, Asmhan was introduced the definition of $\delta^{*}$-open set in Tri-topological spaces [21]. And in [22] she defined the $\delta^{*}$ connectedness in Tri-topological spaces, also Asmhan et al. [23] defined the $\delta^{*}$-base in Tri-topological spaces. In [24], [25] the reader can find a relationships among separation axioms, and a relationships among some types of continuous and open functions in topological, Bitopological and Tritopological spaces, and in 2017, Asmhan introduced the new definitions of countability and separability in Tri-topological spaces namely $\delta^{*}$-countability and $\delta^{*}$-separability [26].

In 2017, Asmhan F.H. presented the concept of the Soft Tritopological spaces [27], and by the same author the concept of Fuzzy Soft topological spaces have been generalized to initiate the study of Fuzzy Soft Tri-topological spaces in [28]. In the present paper, we introduce main relationships among the Fuzzy soft $\tau_{1} \tau_{2} \tau_{3}$-open (closed) set, Fuzzy Soft Tri- $\alpha$ open (closed) set, Fuzzy Soft Tri-pre-open(closed) set and Fuzzy Soft Tri-semi-open (closed) set in Fuzzy Soft Tritopological Spaces.

In section 2 , some preliminary concepts which a central role in our work are given. The main sections of the manuscript is third which the relationships among the Fuzzy soft open (closed) set in Fuzzy Soft Tri-topological Spaces with examples and some theorems are given. Finally in section 5 the conclusions and the idea of future work is suggested.

\section{PRELIMINARIES}

In this section, we present the basic definitions of Fuzzy Soft set theory, Soft set theory and Fuzzy set theory that are useful for subsequent discussions and which will be a central role in our work.

Definition 2.1. [1] Let $\mathcal{U}$ be an universe. A Fuzzy set $X$ over $\mathcal{U}$ is a set defined by a function $\mu_{X}$ representing a mapping $\mu_{X}: \mathcal{U} \rightarrow[0,1], \mu_{X}$ is called the membership function of $X$ and 
the value $\mu_{X}(u)$ is called the grade of membership of $u \in \mathcal{U}$. The value represents the degree of $u$ belonging to the Fuzzy set $X$.Thus, a Fuzzy set $X$ over $\mathcal{U}$ can be represented as follows : $X=\left\{\left(\mu_{X}(u) / u\right): u \in \mathcal{U}, \mu_{X} \in[0,1]\right\}$

Definition 2.2. [4] Let the set $\mathcal{U}$ be an initial universe and $E$ be a set of parameters. Let $\mathcal{P}(\mathcal{U})$ denotes the power set of $\mathcal{U}$ and $\mathbb{A}$ be a non-empty subset of $E$. A pair $(\mathrm{F}, \mathbb{A})$ is said to be a soft-set over $\mathcal{U}$ where $\mathrm{F}$ is a mapping given by $\mathrm{F}: \mathbb{A} \rightarrow$ $\mathcal{P}(\mathcal{U})$.

In other words, a soft-set over $\mathcal{U}$ is a parametrized family of subsets of the universe $\mathcal{U}$. For e $\in \mathbb{A}, \mathrm{F}(\mathrm{e})$ may be considered as the set of e-approximate elements of the soft $-\operatorname{set}(\mathrm{F}, \mathbb{A})$. Clear that, a soft - set is not a set.

Definition 2.3. [29] A Fuzzy Soft set $S_{A}$ over $U$ is a set defined by a function $\xi_{A}$ representing amapping $\xi_{A}: E \rightarrow$ $F(\mathcal{U})$ such that $\xi_{A}(x)=\emptyset$ if $x \notin A$. Here $\xi_{A}$ is called Fuzzy approximate function of the Fuzzy Soft set $S_{A}$ and the value $\xi_{A}(x)$ is a set called $x$ - element of the Fuzzy Soft set for all $x \in E$. Thus, an Fuzzy Soft set $S_{A}$ over $U$ can be represented by the set of ordered pair $S_{A}=\left\{\left(x, \xi_{A}(x): x \in E, \xi_{A}(x) \in\right.\right.$ $F(\mathcal{U})\}$.

Note that the set of all Fuzzy Soft sets over $\mathcal{U}$ will be denoted by F.S.(U) or F.S. $(U, E)$.

Definition 2.4. [7] Let $S_{A} \in$ F.S. $(U, E)$. If $\xi_{A}(x)=\emptyset$ for all $x \in E$, then $S_{A}$ is called an empty Fuzzy Soft set, denoted by $S_{\emptyset}$ or $\left(0_{E}\right)$.

Definition 2.5.[7] Let $S_{A} \in$ F.S. $(\mathcal{U}, E)$.If $\xi_{A}(x)=\mathcal{U}$ for all $x \in A$, then $S_{A}$ is called A- universal Fuzzy Soft set, denoted by $S_{E}$ or $\left(1_{E}\right)$

Definition 2.6. [7] Let $S_{A}, S_{B} \in$ F.S. $(\mathcal{U}, E)$. Then $S_{A}$ is called a Fuzzy Soft subset of $S_{B}$, denoted by $S_{A} \sqsubseteq S_{B}$ If $\xi_{A}(x) \sqsubseteq$ $\xi_{B}(x)$ for all $x \in E$.

Definition 2.7. [30] $S_{A} \subseteq S_{B}$ does not mean as in the classical subset. (i.e. does not imply that every element of $S_{A}$ is an element of $S_{B}$ ).

Definition 2.8. [7] Let $S_{A}, S_{B} \in$ F.S. $(\mathcal{U}, E)$. Then the two Fuzzy Soft sets $S_{A}$ and $S_{B}$ are equal, written as $S_{A}=S_{B}$ If and only if $\xi_{A}(x)=\xi_{B}(x)$ for All $x \in E$.

Definition 2.9. [7] Let $\mathcal{S}_{A} \in$ F.S. $(U, E)$. Then the complement $\mathcal{S}_{A}^{c}$ of $\mathcal{S}_{A}$ is a Fuzzy Soft set such that $\xi_{A^{c}}(x)=$ $\xi_{A}^{c}(x)$ for all $x \in E$, where $\xi_{A}^{c}(x)$ is complement of all set $\xi_{A}(x)$. Clear that $\left(\mathcal{S}_{A}^{c}\right)^{c}=\mathcal{S}_{A}, \mathcal{\delta}_{\emptyset}^{c}=\mathcal{S}_{E}$ and $\mathcal{S}_{E}^{c}=\mathcal{S}_{E}$.

Definition 2.10.[7] Let $S_{A}, S_{B} \in$ F.S. $(\mathcal{U}, E)$. Then the union of $S_{A}$ and $S_{B}$, denoted by $S_{A} \sqcup S_{B}$, is defined by its Fuzzy approximate function $\xi_{A \sqcup B}=\xi_{A}(x) \sqcup \xi_{B}(x)$ for all $x \in E$.

Definition 2.11.[7] Let $S_{A}, S_{B} \in$ F.S. $(\mathcal{U}, E)$. Then the intersection of $S_{A}$ and $S_{B}$, denoted by $\delta_{A} \Pi \mathcal{S}_{B}$, is defined by its Fuzzy approximate function $\xi_{A \sqcap B}=\xi_{A}(x) \sqcap \xi_{B}(x)$ for all $x \in E$.

Definition 2.12. [30] Let $\tau$ be the collection or sub family of Fuzzy Soft set over $\mathcal{U}$ (i.e. $\tau \sqsubseteq$ F. S. $(\mathcal{U}, E)$. Then $\tau$ is said to be a Fuzzy Soft topology on the universal set $\mathcal{U}$ if satisfying the following properties:

(i) $\quad \delta_{\varnothing}, \delta_{E} \in \tau$

(ii) If $\mathcal{S}_{A}, \mathcal{S}_{B} \in \tau$, then $\mathcal{S}_{A} \Pi \mathcal{S}_{B} \in \tau$

(iii) If $\mathcal{S}_{A j} \in \tau, \forall \mathrm{j} \in \Lambda$, where $\Lambda$ is some index set, then $\bigsqcup_{\mathrm{j} \in \Lambda} \delta_{\mathrm{Aj}} \in \tau$.

Then the triple $(\mathcal{U}, E, \tau)$ is called a Fuzzy Soft topological space over $\mathcal{U}$. And each member of $\tau$ is called Fuzzy Soft open set in $(\mathcal{U}, E, \tau)$. Also Fuzzy Soft set is called Fuzzy Soft closed if and only if its complement is Fuzzy Soft open.

Definition 2.13. [16] Let $\left(\mathcal{U}, E, \tau_{1}\right)$ and $\left(\mathcal{U}, E, \tau_{2}\right)$ be the two Fuzzy Soft topological spaces over $\mathcal{U}$. Then $\left(\mathcal{U}, E, \tau_{1}, \tau_{2}\right)$ is called a Fuzzy Soft bitopological space .
Definition 2.14. [28] Let $\left(U, E, \tau_{1}\right),\left(\mathcal{U}, E, \tau_{2}\right)$ and $\left(\mathcal{U}, E, \tau_{3}\right)$ be the three Fuzzy Soft topological spaces on $\mathcal{U}$. Then a space equipped with three Fuzzy Soft topologies, i.e. triple of Fuzzy Soft topologies on the same set is called a Fuzzy Soft Tritopological space and denoted by $\left(\mathcal{U}, E, \tau_{1}, \tau_{2}, \tau_{3}\right)$. Where the three Fuzzy Soft topological space are independently satisfy the axioms of Fuzzy Soft topological space.

Definition 2.15. [28] Let $\left(\mathcal{U}, \mathrm{E}, \tau_{1}, \tau_{2}, \tau_{3}\right)$ be a fuzzy soft tritopological space and $\Gamma_{\mathrm{E}}$ is a fuzzy soft set in $\mathcal{U}$, then $\Gamma_{\mathrm{E}}$ is called a fuzzy soft $\tau_{1} \tau_{2} \tau_{3}$-open set if $\Gamma_{E}=f_{E} \sqcup g_{E} \sqcup h_{E}$, where $f_{E} \in \tau_{1}, g_{E} \in \tau_{2}$ and $h_{E} \in \tau_{3}$. The complement of fuzzy soft $\tau_{1} \tau_{2} \tau_{3}$-open set is called fuzzy soft $\tau_{1} \tau_{2} \tau_{3}$-closed. The family of all fuzzy soft $\tau_{1} \tau_{2} \tau_{3}$-open sets is denoted by FS. $\tau_{1} \tau_{2} \tau_{3} . O(\mathcal{U})$. And the family of all fuzzy soft $\tau_{1} \tau_{2} \tau_{3}$ closed sets is denoted by FS. $\tau_{1} \tau_{2} \tau_{3} . \mathrm{C}(\mathcal{U})$

Definition 2.16. [28] Let $\left(\mathcal{U}, E, \tau_{1}, \tau_{2}, \tau_{3}\right)$ be a fuzzy soft tritopological space, and $\Gamma_{E}$ is a fuzzy soft set in $\mathcal{U}$, then:

i. The fuzzy soft $\tau_{1} \tau_{2} \tau_{3}$-closure of $\Gamma_{E}$ denoted by FS. $\tau_{1} \tau_{2} \tau_{3} c l\left(\Gamma_{E}\right)$ is defined by:

FS. $\tau_{1} \tau_{2} \tau_{3} \operatorname{cl}\left(\Gamma_{E}\right)=\Pi\left\{g_{E}: \Gamma_{E}\right.$ 巨 $g_{E}, \quad$ and $g_{E}$ is fuzzy soft $\tau_{1} \tau_{2} \tau_{3}$-closed

ii. The fuzzy soft $\tau_{1} \tau_{2} \tau_{3}$-interior of $\Gamma_{E}$, denoted by FS. $\tau_{1} \tau_{2} \tau_{3} \operatorname{int}\left(\Gamma_{E}\right)$ is defined by:

FS. $\tau_{1} \tau_{2} \tau_{3} \operatorname{int}\left(\Gamma_{E}\right)=\sqcup\left\{h_{E}: h_{E} \sqsubseteq \Gamma_{E}, \quad\right.$ and $h_{E}$ is

fuzzy soft $\tau_{1} \tau_{2} \tau_{3}$-open

Definition 2.17. [28] Let $\left(\mathcal{U}, \mathrm{E}, \tau_{1}, \tau_{2}, \tau_{3}\right)$ be a Fuzzy Soft Tri-topological space and let $F_{E}$ be a Fuzzy Soft set over $(U, E)$ then $F_{E}$ is called a Fuzzy Soft $\tau_{1} \tau_{2} \tau_{3}$ - $\alpha$-open set (or Fuzzy Soft Tri- $\alpha$-open set) if $F_{E}$ ㄷ F. S. $\tau_{1} \tau_{2} \tau_{3} \operatorname{int}\left(\right.$ F. S. $\tau_{1} \tau_{2} \tau_{3}$ cl(F. S. $\left.\left.\tau_{1} \tau_{2} \tau_{3} \operatorname{int}\left(F_{E}\right)\right)\right)$. The complement of Fuzzy Soft $\tau_{1} \tau_{2} \tau_{3}$ - $\alpha$-open set is defined to be Fuzzy Soft $\tau_{1} \tau_{2} \tau_{3}-\alpha$-closed.

Definition 2.18. [28] Let $\left(\mathcal{U}, \mathrm{E}, \tau_{1}, \tau_{2}, \tau_{3}\right)$ be a Fuzzy Soft Tri-topological space and let $F_{E}$ be a Fuzzy Soft set over $(\mathcal{U}, E) F_{E}$ is referred to as a Fuzzy Soft $\tau_{1} \tau_{2} \tau_{3}$-pre-open set (or Fuzzy Soft Tri-pre-open set) if

$F_{E}$ ㄷ F.S. $\tau_{1} \tau_{2} \tau_{3} \operatorname{int}\left(\right.$ F. S. $\tau_{1} \tau_{2} \tau_{3} \operatorname{cl}\left(\left(F_{E}\right)\right)$. The complement of Fuzzy Soft $\tau_{1} \tau_{2} \tau_{3}$-pre-open set is defined to be Fuzzy Soft $\tau_{1} \tau_{2} \tau_{3}$-pre-closed

Definition 2.19. [28] Let $\left(\mathcal{U}, \mathrm{E}, \tau_{1}, \tau_{2}, \tau_{3}\right)$ be a Fuzzy Soft Tri-topological space and let $F_{E}$ be a Fuzzy Soft set over $(\mathcal{U}, E)$ then $F_{E}$ is called a Fuzzy Soft $\tau_{1} \tau_{2} \tau_{3}$-semi-open set (or Fuzzy Soft Tri-semi-open set) if $F_{E}$ 드 (F.S. $\tau_{1} \tau_{2} \tau_{3}$ cl(F.S. $\tau_{1} \tau_{2} \tau_{3} \operatorname{int}\left(F_{E}\right)$ ). The complement of Fuzzy Soft $\tau_{1} \tau_{2} \tau_{3}$-semi-open set is defined to be Fuzzy Soft $\tau_{1} \tau_{2} \tau_{3}$-semi-closed.

\section{RELATIONSHIPS AMONG THE FUZZY SOFT $\tau_{1} \tau_{2} \tau_{3}$-OPEN SET, FUZZY SOFT TRI- $\alpha$-OPEN SET, FUZZY SOFT TRI-PRE-OPEN SET AND FUZZY SOFT TRI-semi-OPEN SET IN FUZZY SOFT TRI-TOPOLOGICAL SPACES}

1. In this section study of relationships among the Fuzzy Soft open sets in Fuzzy Soft Tri-topological Spaces is initiated by stating the following theorems.

Theorem 3.1. Every Fuzzy Soft $\tau_{1} \tau_{2} \tau_{3}$ open (closed) set in Fuzzy Soft Tri-topological space is Fuzzy Soft $\tau_{1} \tau_{2} \tau_{3}$ -Semi - open (closed).

Proof. let $F_{E}$ is Fuzzy Soft $\tau_{1} \tau_{2} \tau_{3}$ open set, then F.S. $\tau_{1} \tau_{2} \tau_{3} \operatorname{int}\left(F_{E}\right)=F_{E}$. Since 
$F_{E} \sqsubseteq F . S . \tau_{1} \tau_{2} \tau_{3} \operatorname{cl}\left(F_{E}\right)$ by the definition of F.S. $\tau_{1} \tau_{2} \tau_{3}-$ clouser, then $F_{E} \subseteq$ F.S. $\tau_{1} \tau_{2} \tau_{3} \operatorname{cl}\left(F . S . \tau_{1} \tau_{2} \tau_{3} \operatorname{int}\left(F_{E}\right)\right)$. Thus $F_{E}$ is Fuzzy Soft $\tau_{1} \tau_{2} \tau_{3}-S e m i-$ open. And it is easy to prove the closed sets.

Remark 3.2. The converse of theorem 3.1. above is not true in general as shown in the following example.

Example 3.3. Let $U=\left\{u_{1}, u_{2}\right\}, E=\left\{x_{1}, x_{2}\right\}, \tau_{1}=$ $\left\{0_{E}, 1_{E}, \psi_{1 E}, \psi_{2 E}\right\}, \tau_{2}=\left\{0_{E}, 1_{E}, \gamma_{1 E}, \gamma_{2 E}\right\}$ and $\tau_{3}=$ $\left\{0_{E}, 1_{E}, \beta_{E}\right\}$. Where $\psi_{1 E}, \psi_{2 E}, \gamma_{1 E}, \gamma_{2 E}$ and $\beta_{E}$ are Fuzzy Soft sets over $(\mathcal{U}, E)$ defined as follows ;

$\psi_{1 E}=\left\{\left(x_{1},\left\{0.3 / u_{1}, 0.4 / u_{2}\right\}\right),\left(x_{2},\left\{0.1 / u_{1}, 0.2 / u_{2}\right\}\right)\right\}$

$\psi_{2 E}=\left\{\left(x_{1},\left\{0.4 / u_{1}, 0.6 / u_{2}\right\}\right),\left(x_{2},\left\{0.2 / u_{1}, 0.5 / u_{2}\right\}\right)\right\}$

$\gamma_{1 E}=\left\{\left(x_{1},\left\{0.3 / u_{1}, 0.4 / u_{2}\right\}\right),\left(x_{2},\left\{0.3 / u_{1}, 0.8 / u_{2}\right\}\right)\right\}$

$\gamma_{2 E}=\left\{\left(x_{1},\left\{0.2 / u_{1}, 0.4 / u_{2}\right\}\right),\left(x_{2},\left\{0.2 / u_{1}, 0.5 / u_{2}\right\}\right)\right\}$

And $\beta_{E}=\left\{\left(x_{1},\left\{0.3 / u_{1}, 0.0 / u_{2}\right\}\right),\left(x_{2},\left\{0.0 / u_{1}, 0.2 / u_{2}\right\}\right)\right\}$

Then $\tau_{1}, \tau_{2}$ and $\tau_{3}$ are three Fuzzy Soft topologies over

$(\mathcal{U}, \mathrm{E})$. Therefore $\left(\mathcal{U}, E, \tau_{1}, \tau_{2}, \tau_{3}\right)$ is a Fuzzy Soft Tritopological space. It is clear that the family of all Fuzzy Soft $\tau_{1} \tau_{2} \tau_{3^{-}}$open sets are: F.S. $\tau_{1} \tau_{2} \tau_{3} . \mathrm{O}(\mathcal{U})=\left\{0_{E}, 1_{E}, \psi_{1 E}, \psi_{2 E}, \gamma_{1 E}, \gamma_{2 E}, \beta_{E}, \lambda_{E}, \delta_{E}\right\}$ $=\tau_{1} \sqcup \tau_{2} \sqcup \tau_{3} \sqcup\left\{\lambda_{\mathrm{E}}, \delta_{\mathrm{E}}\right\}$. Where $\psi_{2 \mathrm{E}} \sqcup \gamma_{2 \mathrm{E}} \sqcup \beta_{\mathrm{E}}=\psi_{2 \mathrm{E}}$ $\psi_{1 \mathrm{E}} \sqcup \gamma_{1 \mathrm{E}} \sqcup \beta_{\mathrm{E}}=\gamma_{1 \mathrm{E}}$

$\psi_{2 E} \sqcup \gamma_{1 E} \sqcup \beta_{E}=\lambda_{E}=\left\{\left(x_{1},\left\{0.4 / u_{1}, 0.6 / u_{2}\right\}\right),\left(x_{2},\{0.3\right.\right.$

$\psi_{1 E} \sqcup \gamma_{2 E} \sqcup \beta_{E}=\delta_{E}$ $\left.\left.\left./ u_{1}, 0.8 / u_{2}\right\}\right)\right\}$

$=\left\{\left(x_{1},\left\{0.3 / u_{1}, 0.4 / u_{2}\right\}\right),\left(x_{2},\{0.2\right.\right.$ $\left.\left.\left./ u_{1}, 0.5 / u_{2}\right\}\right)\right\}$

Now, we find the Fuzzy Soft $\tau_{1} \tau_{2} \tau_{3}$ closed sets :

F.s. $\tau_{1} \tau_{2} \tau_{3} \quad . C(\mathcal{U})=\left\{1_{\mathrm{E}}, 0_{\mathrm{E}}, \psi_{1 \mathrm{E}}^{\mathrm{c}}, \psi_{2 \mathrm{E}}^{\mathrm{c}}, \gamma_{1 \mathrm{E}}^{\mathrm{c}}, \gamma_{2 \mathrm{E}}^{\mathrm{c}}, \beta_{\mathrm{E}}^{\mathrm{c}}, \lambda_{\mathrm{E}}^{\mathrm{c}}, \delta_{\mathrm{E}}^{\mathrm{c}}\right\}$, where defined as follows;

$\left\{\left(x_{1},\left\{0.7 / u_{1}, 0.6 / u_{2}\right\}\right),\left(x_{2},\left\{0.9 / u_{1}, 0.8 / u_{2}\right\}\right)\right\}$ $\psi_{1 E}^{c}=$

$\psi_{2 E}^{c}=\left\{\left(x_{1},\left\{0.6 / u_{1}, 0.4 / u_{2}\right\}\right),\left(x_{2},\left\{0.8 / u_{1}, 0.5 / u_{2}\right\}\right)\right\}$

$\gamma_{1 E}^{c}=\left\{\left(x_{1},\left\{0.7 / u_{1}, 0.6 / u_{2}\right\}\right),\left(x_{2},\left\{0.7 / u_{1}, 0.2 / u_{2}\right\}\right)\right\}$

$\gamma_{2 E}^{c}=\left\{\left(x_{1},\left\{0.8 / u_{1}, 0.6 / u_{2}\right\}\right),\left(x_{2},\left\{0.8 / u_{1}, 0.5 / u_{2}\right\}\right)\right\}$

$\beta_{E}^{c}=\left\{\left(x_{1},\left\{0.7 / u_{1}, 1.0 / u_{2}\right\}\right),\left(x_{2},\left\{1.0 / u_{1}, 0.8 / u_{2}\right\}\right)\right\}$

$\lambda_{E}^{c}=\left\{\left(x_{1},\left\{0.6 / u_{1}, 0.4 / u_{2}\right\}\right),\left(x_{2},\left\{0.7 / u_{1}, 0.2 / u_{2}\right\}\right)\right\}$

$\delta_{E}^{c}=\left\{\left(x_{1},\left\{0.7 / u_{1}, 0.6 / u_{2}\right\}\right),\left(x_{2},\left\{0.8 / u_{1}, 0.5 / u_{2}\right\}\right)\right\}$

If we take the Fuzzy Soft set $D_{E}$ which defined as; $\left\{\left(x_{1},\{0.3 /\right.\right.$ $\left.\left.u_{1}, 0.1 / u_{2}\right\}\right),\left(x_{2},\left\{0.1 / u_{1}, 0.2 / u_{2}\right\}\right.$, then $D_{E}$ is a Fuzzy Soft $\tau_{1} \tau_{2} \tau_{3}$-semi-open set since

F.S. $\tau_{1} \tau_{2} \tau_{3} \operatorname{int}\left(D_{E}\right)=\left\{\beta_{E} \sqcup 0_{E}\right\}=\beta_{E}$

F.S. $\tau_{1} \tau_{2} \tau_{3} \operatorname{cl}\left(\beta_{E}\right)=\lambda_{\mathrm{E}}^{\mathrm{c}}$, thus

F.S. $\tau_{1} \tau_{2} \tau_{3} c l\left(F . S . \tau_{1} \tau_{2} \tau_{3} \operatorname{int}\left(D_{E}\right)\right)=\lambda_{E}^{c}$ hence $D_{E}$ 드 $\lambda_{E}^{c}$ but the Fuzzy Soft set $D_{E}$ is not Fuzzy Soft $\tau_{1} \tau_{2} \tau_{3}$ open set.

Theorem 3.4. Every Fuzzy soft $\tau_{1} \tau_{2} \tau_{3}$ open (closed) set in Fuzzy Soft Tri-topological space is Fuzzy Soft $\tau_{1} \tau_{2} \tau_{3}$ -pre - open (closed).

Proof. let $F_{E}$ is Fuzzy Soft $\tau_{1} \tau_{2} \tau_{3}$-open set, then F.S. $\tau_{1} \tau_{2} \tau_{3} \operatorname{int}\left(F_{E}\right)=F_{E}$. Since $F_{E} \subseteq F . S . \tau_{1} \tau_{2} \tau_{3} \operatorname{cl}\left(F_{E}\right)$ then $F_{E} \sqsubseteq$ F.S. $\tau_{1} \tau_{2} \tau_{3} \operatorname{int}\left(F . S . \tau_{1} \tau_{2} \tau_{3} c l\left(F_{E}\right)\right)$. Thus $F_{E}$ is Fuzzy Soft $\tau_{1} \tau_{2} \tau_{3}-$ pre - open. And it is easy to prove the closed sets.

Remark 3.5. The converse of theorem 3.4 above is not true in general as shown in the following example.

Example 3.6. In example 3.3, if we consider the Fuzzy Soft set $D_{E}$ in which can be described as; $\left\{\left(x_{1},\left\{0.3 / u_{1}, 0.1 / u_{2}\right\}\right)\right.$, $\left(x_{2},\left\{0.1 / u_{1}, 0.2 / u_{2}\right\}\right.$, then $D_{E}$ is a Fuzzy Soft $\tau_{1} \tau_{2} \tau_{3}$-preopen set since $=\beta_{E}$
F.S. $\tau_{1} \tau_{2} \tau_{3} \operatorname{cl}\left(D_{E}\right)=$

$\left\{\psi_{1 E}^{c} \Pi \psi_{2 E}^{c} \Pi \gamma_{1 E}^{c} \Pi \gamma_{2 E}^{c} \Pi \beta_{E}^{c} \Pi \lambda_{E}^{c} \Pi \delta_{E}^{c} \Pi 1_{E}\right\}=\lambda_{\mathrm{E}}^{\mathrm{c}}$,

then F.S. $\tau_{1} \tau_{2} \tau_{3} \operatorname{int}\left(\lambda_{\mathrm{E}}^{\mathrm{c}}\right)=\left\{\psi_{1 E} \sqcup \beta_{E} \sqcup 0_{E}\right\}=\psi_{1 E}$ thus

F.S. $\tau_{1} \tau_{2} \tau_{3} \operatorname{int}\left(F . S . \tau_{1} \tau_{2} \tau_{3} \operatorname{cl}\left(\left(D_{E}\right)\right)\right)=\psi_{1 E}$ hence $D_{E}$ 드 $\psi_{1 E}$

but the Fuzzy Soft set $D_{E}$ is not Fuzzy Soft $\tau_{1} \tau_{2} \tau_{3}$-open set.

Theorem 3.7. Every Fuzzy soft $\tau_{1} \tau_{2} \tau_{3}$-open (closed) set in Fuzzy Soft Tri-topological space is Fuzzy Soft $\tau_{1} \tau_{2} \tau_{3}-\alpha-$ open (closed).

Proof. let $F_{E}$ be a Fuzzy Soft $\tau_{1} \tau_{2} \tau_{3}$ open set, then F.S. $\tau_{1} \tau_{2} \tau_{3} \operatorname{int}\left(F_{E}\right)=F_{E}$. Since by theorem 3.1 ( Every Fuzzy soft $\tau_{1} \tau_{2} \tau_{3}$-open (closed) set in Fuzzy Soft Tritopological space is Fuzzy Soft $\tau_{1} \tau_{2} \tau_{3}-$ Semi-open (closed)), then $F_{E} \subseteq F . S . \tau_{1} \tau_{2} \tau_{3} \operatorname{int}\left(F . S . \tau_{1} \tau_{2} \tau_{3} \operatorname{cl}\left(\left(F_{E}\right)\right)\right.$ and by F.S. $\tau_{1} \tau_{2} \tau_{3} \operatorname{int}\left(F_{E}\right)=F_{E}$. Thus

$F_{E} \subseteq$ F.S. $\tau_{1} \tau_{2} \tau_{3} \operatorname{int}\left(F . S . \tau_{1} \tau_{2} \tau_{3} \operatorname{cl}\left(\right.\right.$ F.S. $\left.\tau_{1} \tau_{2} \tau_{3} \operatorname{int}\left(F_{E}\right)\right)$.

Hence $F_{E}$ is Fuzzy Soft $\tau_{1} \tau_{2} \tau_{3}-\alpha-$ open. And it is easy to prove the closed sets.

Remark 3.8. The converse of theorem 3.7 above is not true in general as shown in the following example.

Example 3.9. In example 3.3, if we consider the Fuzzy Soft set $D_{E}$ in which can be described as; $\left\{\left(x_{1},\left\{0.3 / u_{1}, 0.1 / u_{2}\right\}\right)\right.$, $\left(x_{2},\left\{0.1 / u_{1}, 0.2 / u_{2}\right\}\right.$, then $D_{E}$ is a Fuzzy Soft $\tau_{1} \tau_{2} \tau_{3}-\alpha-$ open set since

F.S. $\tau_{1} \tau_{2} \tau_{3} \operatorname{int}\left(D_{E}\right)=\left\{\beta_{E} \sqcup 0_{E}\right\}=\beta_{E}$

F.S. $\tau_{1} \tau_{2} \tau_{3} \operatorname{cl}\left(\beta_{E}\right)=$

$\left\{\psi_{1 E}^{c} \Pi \psi_{2 E}^{c} \Pi \gamma_{1 E}^{c} \Pi \gamma_{2 E}^{c} \Pi \beta_{E}^{c} \Pi \lambda_{\mathrm{E}}^{\mathrm{c}} \Pi \delta_{E}^{c} \Pi 1_{E}\right\}=\lambda_{\mathrm{E}}^{\mathrm{c}}$,

then F.S. $\tau_{1} \tau_{2} \tau_{3} \operatorname{int}\left(\lambda_{\mathrm{E}}^{\mathrm{c}}\right)=\left\{\psi_{1 E} \sqcup \beta_{E} \sqcup 0_{E}\right\}=\psi_{1 E}$ thus

F.S. $\tau_{1} \tau_{2} \tau_{3} \operatorname{int}\left(F . S . \tau_{1} \tau_{2} \tau_{3} \operatorname{cl}\left(F . S . \tau_{1} \tau_{2} \tau_{3} \operatorname{int}\left(D_{E}\right)\right)\right)=\psi_{1 E}$ hence $D_{E} \sqsubseteq \psi_{1 E}$

but the Fuzzy Soft set $D_{E}$ is not Fuzzy Soft $\tau_{1} \tau_{2} \tau_{3}$-open set.

Theorem 3.10. Every Fuzzy soft $\tau_{1} \tau_{2} \tau_{3}-\alpha$-open (closed) in Fuzzy Soft Tri-topological space is Fuzzy soft $\tau_{1} \tau_{2} \tau_{3}$ - semi - open (closed).

Proof. To prove that Fuzzy soft $\tau_{1} \tau_{2} \tau_{3}-\alpha$-open (closed) $\Rightarrow$ Fuzzy soft $\tau_{1} \tau_{2} \tau_{3}$-semi open (closed), let $F_{E}$ be a Fuzzy soft $\tau_{1} \tau_{2} \tau_{3}-\alpha$-open set, $\quad$ since $F_{E} \subseteq$ F.S. $\tau_{1} \tau_{2} \tau_{3} \operatorname{cl}\left(F_{E}\right)$ and by the definition $F_{E}$ F.S. $\tau_{1} \tau_{2} \tau_{3} \operatorname{int}\left(F . S . \tau_{1} \tau_{2} \tau_{3} \operatorname{cl}\left(\right.\right.$ F.S. $\left.\tau_{1} \tau_{2} \tau_{3} \operatorname{int}\left(F_{E}\right)\right)$, then $F_{E} \subseteq F . S . \tau_{1} \tau_{2} \tau_{3} \operatorname{cl}\left(F . S . \tau_{1} \tau_{2} \tau_{3} \operatorname{int}\left(F_{E}\right)\right)$. Hence $F_{E}$ is a Fuzzy Soft $\tau_{1} \tau_{2} \tau_{3}$-semi-open set.

Remark 3.11. The converse of theorem 3.10 above is not true in general as shown in the following example.

Example 3.12. In example 3.3, if we take the Fuzzy Soft set $x_{E}$ which defined as; $\left\{\left(x_{1},\left\{0.4 / u_{1}, 0.2 / u_{2}\right\}\right),\left(x_{2},\{0.3 /\right.\right.$ $\left.u_{1}, 0.2 / u_{2}\right\}$, then $x_{E}$ is a Fuzzy Soft $\tau_{1} \tau_{2} \tau_{3}$-semi-open set since $\quad$ F.S. $\tau_{1} \tau_{2} \tau_{3} \operatorname{int}\left(x_{E}\right)=\left\{\beta_{E} \sqcup 0_{E}\right\}=\quad \beta_{E}$ F.S. $\tau_{1} \tau_{2} \tau_{3} \operatorname{cl}\left(\beta_{E}\right)=\lambda_{\mathrm{E}}^{\mathrm{c}} \quad, \quad$ thus F.S. $\tau_{1} \tau_{2} \tau_{3} \operatorname{cl}\left(F . S . \tau_{1} \tau_{2} \tau_{3} \operatorname{int}\left(x_{E}\right)\right)=\lambda_{E}^{c}$, hence $x_{E} \sqsubseteq \lambda_{E}^{c}$, but $x_{E}$ is not a Fuzzy Soft $\tau_{1} \tau_{2} \tau_{3}-\alpha$-open set, since F.S. $\tau_{1} \tau_{2} \tau_{3} \operatorname{int}\left(x_{E}\right)=\left\{\beta_{E} \sqcup 0_{E}\right\}=\beta_{E}$

F.S. $\tau_{1} \tau_{2} \tau_{3} c l\left(\beta_{E}\right)=\lambda_{\mathrm{E}}^{\mathrm{c}}$

F.S. $\tau_{1} \tau_{2} \tau_{3} \operatorname{int}\left(\lambda_{\mathrm{E}}^{\mathrm{c}}\right)=\left\{\beta_{E} \sqcup 0_{E} \sqcup \psi_{1 E}\right\}=\psi_{1 E}$ F.S. $\tau_{1} \tau_{2} \tau_{3} \operatorname{int}\left(F . S . \tau_{1} \tau_{2} \tau_{3} \operatorname{cl}\left(F . S . \tau_{1} \tau_{2} \tau_{3} \operatorname{int}\left(x_{E}\right)\right)\right)=$ $\psi_{1 E}$, thus $x_{E} \nsubseteq \psi_{1 E}$ 
Theorem 3.13. Every Fuzzy soft $\tau_{1} \tau_{2} \tau_{3}-\alpha$-open (closed) in Fuzzy Soft Tri-topological space is Fuzzy soft $\tau_{1} \tau_{2} \tau_{3}-$ preopen (closed).

Proof. let $F_{E}$ be a Fuzzy Soft $\tau_{1} \tau_{2} \tau_{3}-\alpha$-open set then by the definition

$F_{E}$ F.S. $\tau_{1} \tau_{2} \tau_{3} \operatorname{int}\left(F . S . \tau_{1} \tau_{2} \tau_{3} \operatorname{cl}\left(\right.\right.$ F.S. $\left.\tau_{1} \tau_{2} \tau_{3} \operatorname{int}\left(F_{E}\right)\right)$

then $F_{E} \subseteq F . S . \tau_{1} \tau_{2} \tau_{3} \operatorname{int}\left(F . S . \tau_{1} \tau_{2} \tau_{3} c l\left(F_{E}\right)\right)$. Hence $F_{E}$ is a Fuzzy Soft $\tau_{1} \tau_{2} \tau_{3}$-pre -open.

Remark 3.14. The converse of theorem 3.13 above is not true in general as shown in the following example.

Example 3.15. In example 3.3, if we take the Fuzzy Soft set $\mathrm{z}_{\mathrm{E}} \quad$ which defined as; $\left\{\left(x_{1},\left\{0.2 / u_{1}, 0.3 / u_{2}\right\}\right),\left(x_{2},\{0.3 /\right.\right.$ $\left.u_{1}, 0.7 / u_{2}\right\}$, then $z_{E}$ is a Fuzzy Soft $\tau_{1} \tau_{2} \tau_{3}$-pre -open since

F.S. $\tau_{1} \tau_{2} \tau_{3} \operatorname{cl}\left(\mathrm{z}_{\mathrm{E}}\right)=\left\{\psi_{1 E}^{c} \Pi \beta_{E} \Pi 1_{E}\right\}=\psi_{1 E}^{c}$

F.S. $\tau_{1} \tau_{2} \tau_{3} \operatorname{int}\left(\psi_{1 E}^{c}\right)=$

$\left\{\psi_{1 E} \sqcup \psi_{2 E} \sqcup \gamma_{1 E} \sqcup \gamma_{2 E} \sqcup \beta_{E} \sqcup \lambda_{E} \sqcup \delta_{E} \sqcup 0_{E}\right\}=\lambda_{E}$, thus

F.S. $\tau_{1} \tau_{2} \tau_{3} \operatorname{int}\left(F . S . \tau_{1} \tau_{2} \tau_{3} \operatorname{cl}\left(\left(z_{E}\right)\right)\right)=\lambda_{E}$, hence $z_{E}$ 드 $\lambda_{E}$ but the Fuzzy Soft set $z_{E}$ is not a Fuzzy Soft $\tau_{1} \tau_{2} \tau_{3}-$ $\alpha$-open set since

F.S. $\tau_{1} \tau_{2} \tau_{3} \operatorname{int}\left(z_{E}\right)=\left\{\beta_{E} \sqcup 0_{E}\right\}=\beta_{E} \quad, \quad$ then

F.S. $\tau_{1} \tau_{2} \tau_{3} \operatorname{cl}\left(\beta_{E}\right)=\lambda_{\mathrm{E}}^{\mathrm{c}}$

F.S. $\tau_{1} \tau_{2} \tau_{3} \operatorname{int}\left(\lambda_{\mathrm{E}}^{\mathrm{c}}\right)=\left\{\psi_{1 E} \sqcup 0_{E}\right\}=\psi_{1 E}$.

Thus F.S. $\tau_{1} \tau_{2} \tau_{3} \operatorname{int}\left(F . S . \tau_{1} \tau_{2} \tau_{3} \operatorname{cl}\left(\right.\right.$ F.S. $\left.\left.\tau_{1} \tau_{2} \tau_{3} \operatorname{int}\left(z_{E}\right)\right)\right)=$ $\psi_{1 E}$, hence $z_{E} \nsubseteq \psi_{1 E}$

Theorem 3.16. let $\left(\mathcal{U}, \mathrm{E}, \tau_{1}, \tau_{2}, \tau_{3}\right)$ be a Fuzzy Soft Tritopological space and $F_{E}$ is Fuzzy soft set, then $F_{E}$ is Fuzzy Soft $\tau_{1} \tau_{2} \tau_{3}-S e m i$-open if and only if F.S. $\tau_{1} \tau_{2} \tau_{3} \operatorname{cl}\left(F_{E}\right)$ $=$ F.S. $\tau_{1} \tau_{2} \tau_{3} \operatorname{cl}\left(\right.$ F.S. $\left.\tau_{1} \tau_{2} \tau_{3} \operatorname{int}\left(F_{E}\right)\right)$. Proof. Immediate.

Theorem 3.17. let $\left(\mathcal{U}, \mathrm{E}, \tau_{1}, \tau_{2}, \tau_{3}\right)$ be a Fuzzy Soft Tritopological space and $F_{E}$ is Fuzzy soft, then $F_{E}$ is Fuzzy Soft $\tau_{1} \tau_{2} \tau_{3}-$ Pre-open if and only if F.S. $\tau_{1} \tau_{2} \tau_{3} \operatorname{cl}\left(F_{E}\right)$ $=$ F.S. $\tau_{1} \tau_{2} \tau_{3} \operatorname{int}\left(\right.$ F.S. $\left.\tau_{1} \tau_{2} \tau_{3} c l\left(F_{E}\right)\right)$.

Proof. Immediate.

Theorem 3.18. let $\left(\mathcal{U}, E, \tau_{1}, \tau_{2}, \tau_{3}\right)$ be a Fuzzy Soft Tritopological space over $(U, E)$ and $F_{E}$ be a Fuzzy Soft set over $(\mathcal{U}, \mathrm{E})$. Then $F_{E}$ is Fuzzy Soft $\tau_{1} \tau_{2} \tau_{3}-\alpha$-open if and only if it is a Fuzzy Soft $\tau_{1} \tau_{2} \tau_{3}-$ semi-open set and Fuzzy Soft $\tau_{1} \tau_{2} \tau_{3}-$ pre -open set.

Proof. The necessity follows from theorems 3.10 and 3.13 . For sufficiency, suppose that $F_{E}$ is a Fuzzy soft $\tau_{1} \tau_{2} \tau_{3}-$ semi-open and Fuzzy soft $\tau_{1} \tau_{2} \tau_{3}-$ pre -open set, since is a Fuzzy soft $\tau_{1} \tau_{2} \tau_{3}-$ semi-open and by the definition follows that; $F_{E} \subseteq$ F.S. $\tau_{1} \tau_{2} \tau_{3} c l\left(F . S . \tau_{1} \tau_{2} \tau_{3} \operatorname{int}\left(F_{E}\right)\right)$, this implies that

F.S. $\tau_{1} \tau_{2} \tau_{3} \operatorname{cl}\left(F_{E}\right) \sqsubseteq$

F.S. $\tau_{1} \tau_{2} \tau_{3} \operatorname{cl}\left(F . S . \tau_{1} \tau_{2} \tau_{3} \operatorname{cl}\left(F . S . \tau_{1} \tau_{2} \tau_{3} \operatorname{int}\left(F_{E}\right)\right)\right)=$

F.S. $\tau_{1} \tau_{2} \tau_{3} \operatorname{cl}\left(\right.$ F.S. $\left.\tau_{1} \tau_{2} \tau_{3} \operatorname{int}\left(F_{E}\right)\right)$

therefore,

F.S. $\tau_{1} \tau_{2} \tau_{3} \operatorname{int}\left(F . S . \tau_{1} \tau_{2} \tau_{3} \operatorname{cl}\left(F_{E}\right)\right)$

F.S. $\tau_{1} \tau_{2} \tau_{3} \operatorname{int}\left(F . S . \tau_{1} \tau_{2} \tau_{3} \operatorname{cl}\left(F . S . \tau_{1} \tau_{2} \tau_{3} \operatorname{int}\left(F_{E}\right)\right)\right.$, since $F_{E}$ is Fuzzy soft $\tau_{1} \tau_{2} \tau_{3}-$ pre - open set, then

$F_{E} \sqsubseteq$ F.S. $\tau_{1} \tau_{2} \tau_{3} \operatorname{int}\left(F . S . \tau_{1} \tau_{2} \tau_{3} c l\left(F_{E}\right)\right)$ which follows that

$F_{E} \sqsubseteq$ F.S. $\left.\tau_{1} \tau_{2} \tau_{3} \operatorname{int}\left(F . S . \tau_{1} \tau_{2} \tau_{3} \operatorname{cl}\left(F . S . \tau_{1} \tau_{2} \tau_{3} \operatorname{int}\left(F_{E}\right)\right)\right)\right)$, this completes the proof.

Theorem 3.19. let $F_{E}$ and $G_{E}$ be a Fuzzy Soft sets in Fuzzy Soft Tri-topological space $\left(\mathcal{U}, E, \tau_{1}, \tau_{2}, \tau_{3}\right)$ over $(\mathcal{U}, E)$. Then $F_{E}$ is a Fuzzy Soft $\tau_{1} \tau_{2} \tau_{3}-$ semi-open with $F_{E} \sqsubseteq G_{E}$ ㄷ
F.S. $\tau_{1} \tau_{2} \tau_{3} \operatorname{int}\left(F . S . \tau_{1} \tau_{2} \tau_{3} \operatorname{cl}\left(F_{E}\right)\right)$, then $G_{E}$ is a Fuzzy Soft $\tau_{1} \tau_{2} \tau_{3}-\alpha$-open.

Proof. Suppose that $F_{E}$ is a Fuzzy Soft $\tau_{1} \tau_{2} \tau_{3}-$ semi-open, this implies that $F_{E} \subseteq F . S . \tau_{1} \tau_{2} \tau_{3} \operatorname{cl}\left(F . S . \tau_{1} \tau_{2} \tau_{3} \operatorname{int}\left(F_{E}\right)\right)$. This follows that $G_{E}$ F.S. $\tau_{1} \tau_{2} \tau_{3} \operatorname{int}\left(F . S . \tau_{1} \tau_{2} \tau_{3} \operatorname{cl}\left(F_{E}\right)\right)$ 드 $\left(\right.$ F.S. $\tau_{1} \tau_{2} \tau_{3} \operatorname{int}\left(F . S . \tau_{1} \tau_{2} \tau_{3} \operatorname{cl}\left(F . S . \tau_{1} \tau_{2} \tau_{3} \operatorname{cl}\left(F . S . \tau_{1} \tau_{2} \tau_{3} \operatorname{int}\left(F_{E}\right)\right)\right)\right)=$ F.S. $\tau_{1} \tau_{2} \tau_{3} \operatorname{int}\left(F . S . \tau_{1} \tau_{2} \tau_{3} c l\left(F . S . \tau_{1} \tau_{2} \tau_{3} \operatorname{int}\left(F_{E}\right)\right) \sqsubseteq\right.$ F.S. $\tau_{1} \tau_{2} \tau_{3} \operatorname{int}\left(F . S . \tau_{1} \tau_{2} \tau_{3} \operatorname{cl}\left(F . S . \tau_{1} \tau_{2} \tau_{3} \operatorname{int}\left(G_{E}\right)\right)\right.$. Hence the proof .

Theorem 3.20. let $F_{E}$ and $G_{E}$ be a Fuzzy soft sets in Fuzzy soft Tri-topological space $\left(\mathcal{U}, \mathrm{E}, \tau_{1}, \tau_{2}, \tau_{3}\right)$ over $(\mathcal{U}, \mathrm{E})$. Then $F_{E}$ is a Fuzzy soft $\tau_{1} \tau_{2} \tau_{3}-$ pre-open set if and only if there exists a Fuzzy soft $\tau_{1} \tau_{2} \tau_{3}$-open $G_{E}$ such that $F_{E} \sqsubseteq G_{E}$ 드 F.S. $\tau_{1} \tau_{2} \tau_{3} \operatorname{cl}\left(F_{E}\right)$.

Proof. Suppose that $F_{E}$ is Fuzzy soft $\tau_{1} \tau_{2} \tau_{3}-$ pre-open open set. This imples that $F_{E} \sqsubseteq$ F.S. $\tau_{1} \tau_{2} \tau_{3} \operatorname{int}\left(F . S . \tau_{1} \tau_{2} \tau_{3} c l\left(F_{E}\right)\right) . \quad$ Take $G_{E}=$ F.S. $\tau_{1} \tau_{2} \tau_{3} \operatorname{int}\left(F . S . \tau_{1} \tau_{2} \tau_{3} c l\left(F_{E}\right)\right)$. Then $G_{E}$ is Fuzzy soft $\tau_{1} \tau_{2} \tau_{3}$-open set and $F_{E} \subseteq G_{E} \subseteq$ F.S. $\tau_{1} \tau_{2} \tau_{3} c l\left(F_{E}\right)$.

Conversely, suppose that $F_{E}$ is Fuzzy soft $\tau_{1} \tau_{2} \tau_{3}-$ pre open set with $F_{E} \subseteq G_{E} \subseteq F . S . \tau_{1} \tau_{2} \tau_{3} \operatorname{cl}\left(F_{E}\right)$. We prove that $F_{E}$ is a Fuzzy soft $\tau_{1} \tau_{2} \tau_{3}$-pre-open set. our supposition follows

$F_{E} \sqsubseteq F . S . \tau_{1} \tau_{2} \tau_{3} \operatorname{int}\left(G_{E}\right) \sqsubseteq$

(F.S. $\tau_{1} \tau_{2} \tau_{3} \operatorname{int}\left(F . S . \tau_{1} \tau_{2} \tau_{3} \operatorname{cl}\left(F_{E}\right)\right)$. this implies $F_{E}$ is a Fuzzy soft $\tau_{1} \tau_{2} \tau_{3}-$ pre-open. Hence the proof.

Theorem 3.21. let $F_{E}$ and $G_{E}$ be a Fuzzy Soft sets in Fuzzy Soft Tri-topological space $\left(\mathcal{U}, \mathrm{E}, \tau_{1}, \tau_{2}, \tau_{3}\right)$ over $(\mathcal{U}, \mathrm{E})$. Then $F_{E}$ is a Fuzzy Soft $\tau_{1} \tau_{2} \tau_{3}-$ pre-closed set if and only if there exists a Fuzzy Soft $\tau_{1} \tau_{2} \tau_{3}$-closed $G_{E}$ such that F.S. $\tau_{1} \tau_{2} \tau_{3} \operatorname{int}\left(F_{E}\right) \sqsubseteq G_{E} \sqsubseteq F_{E}$.

Proof. It is easy by using the previous theorem.

Using theorems 3.19 and 3.20 we have the following theorem. Theorem 3.22. let $F_{E}$ and $G_{E}$ be a Fuzzy Soft sets in Fuzzy Soft Tri-topological space $\left(\mathcal{U}, \mathrm{E}, \tau_{1}, \tau_{2}, \tau_{3}\right)$ over $(\mathcal{U}, \mathrm{E})$. Then $F_{E}$ is a Fuzzy Soft $\tau_{1} \tau_{2} \tau_{3}-$ pre-open set if and only if $\left(F_{E}\right)^{C}$ is a Fuzzy Soft $\tau_{1} \tau_{2} \tau_{3}-$ pre-closed set.

\section{CONCLUSIONS}

In the present work, we analyze the relationships among the Fuzzy soft $\tau_{1} \tau_{2} \tau_{3}$-open (closed) set, Fuzzy Soft Tri- $\alpha$-open (closed) set, Fuzzy Soft Tri-pre-open(closed) set and Fuzzy Soft Tri-semi-open (closed) set in Fuzzy Soft Tri-topological Spaces. It is observed that, Fuzzy soft $\tau_{1} \tau_{2} \tau_{3}$ open (closed) $\Rightarrow$ Fuzzy soft $\tau_{1} \tau_{2} \tau_{3}-\alpha$-open (closed) $\Rightarrow$ Fuzzy soft $\tau_{1} \tau_{2} \tau_{3}$ - semi-open (closed). And Fuzzy soft $\tau_{1} \tau_{2} \tau_{3}$-open (closed) Fuzzy $\Rightarrow$ soft $\tau_{1} \tau_{2} \tau_{3}-\alpha-$ open (closed) $\Rightarrow$ Fuzzy soft $\tau_{1} \tau_{2} \tau_{3}$ -pre - open (closed). It is shown by counter examples that converse is not true in general. In particular, we proved that the Fuzzy soft set in a Fuzzy Soft Tri-topological Spaces is Fuzzy soft $\tau_{1} \tau_{2} \tau_{3}-\alpha-$ open if and only if it is Fuzzy soft $\tau_{1} \tau_{2} \tau_{3}$-pre-open and Fuzzy soft $\tau_{1} \tau_{2} \tau_{3}$-semi-open. The applications of fuzzy soft open sets in in a Fuzzy Soft Tritopological Spaces as well as the problems of decision making may be further explored in the future study.

\section{REFERENCES}

[1] L.A.Zadeh, Fuzzy sets, Inform. and Control, 8 (1965) 338-353. 
[2] C. C. Chou, J. M. Yih, J. F. Ding, TC Han, YH Lim, LJ Liu and WK Hsu. Application of a fuzzy EOQ model to the stock management in the manufacture system. Key Engineering Materials. (2012) 361-365.

[3] C. Eksin, G. uzelkaya M, E. Yesil and I. Eksin. Fuzzy logic approach to mimic decision making behaviour of humans in stock management game. Proceedings of the 2008 System Dynamics Conference; 2008.

[4] D. Molodtsov, Soft set theory-first results. Compu. Math. Appl.(37),(1999) 19-31.

[5] P.K. Maji, A. R. Roy and R. Biswas, An application of soft sets in a decision making problem, Comput. Math. Appl. 44 (2002) 1077-1083.

[6] D. Pie and D. Miao, From soft sets to information systems, Granu. comput. IEEE Inter. Conf. 2 (2005) 617-621.

[7] P. Maji, R. Biswas, A. Roy, Fuzzy soft sets, J. Fuzzy Math., 9(3), (2001) 589-602.

[8] A. R. Roy and P. K. Maji, A fuzzy soft set theoretic approach to decision making problems, J. Comput. Appl. Math., 203, (2007) 412-418.

[9] H. Aktas. and N. Cagman, Soft sets and soft groups, Information Sciences, 177, (2007) 2726-2735.

[10] X. Yang, D. Yu, J. Yang and C. Wu, Generalization of soft set theory: from crisp to fuzzy case, In: Bing-Yuan Cao ,eds., Fuzzy Information and Engineering: Proceedings of ICFIE- 2007, Advances in Soft Computing 40, Springer, (2007) 345-355.

[11] X. Yang, T. Y. Lin, J. Yang, Y. Li and D. Yu, Combination of interval-valued fuzzy set and soft set, Comput. Math. Appl., 58, (2009) 521-527.

[12] J.C. Kelly, Bitopological Spaces, proc. London Math. Soc; 13, (1963) 71-83.

[13] I. L. Reilly, On bitopological separation properties. Nanta Math.29, (1972) 14-25.

[14] C. W. Patty, Bitopological spaces. Duke Math. J., 34, (1967) 387-392.

[15] B. Ittanagi, Soft Bitopological Spaces, Comp. \& Math. with App.,107, (2014) 1-4.

[16] P. Mukherjee and C. Park, On fuzzy soft bitopological spaces, Mathematics and Computer Sciences Journal (MCSJ).,10(7), (2015) 1-8.
[17] A. F. Sayed, On Characterizations of Some Types of Fuzzy Soft Sets in Fuzzy Soft Bitopological Spaces, Journal of Advances in Mathematics and Computer Science, 24(3), (2017) 1-12.

[18] A. F. Sayed, some separation axioms in fuzzy soft bitopological spaces, J. Math. Comput. Sci. 8(1), (2018) $28-45$

[19] M. Kovar, On 3-Topological version of Thet- Reularity, Internat. J. Matj, Sci., 23, (2000) 393- 398.

[20] S. Palaniammal , Study of Tri topological spaces, Ph.D Thesis. (2011).

[21] Asmhan Flieh Hassan, $\delta^{*}$-open set in tritopological spaces, M.Sc. thesis, Kufa University. (2004)

[22] Asmhan Flieh Hassan, $\delta^{*}$-connectedness in tritopological space, journal of Thi-Qar university, 6(3), (2011) 20-28.

[23] Asmhan Flieh Hassan, Neeran T. A. \& Alyaa Y., $\delta^{*}$-Base in tritopological space, journal of Kerbala university, 9(3), (2011) $344-352$

[24] Asmhan Flieh Hassan \& Hanan A.H., A relations among the separation axioms in Topological, Bitopological and Tritopological Spaces, journal of Kerbala university, 5(2), (2007) $155-158$

[25] Asmhan Flieh Hassan, A relations among some kinds of continuous and open functions in Topological, Bitopological and Tritopological Spaces, journal of Basrah Researches(sciences), 35(4), (2009) 1-4.

[26] Asmhan Flieh Hassan, Countability and Separability in Tritopological Spaces ( $\delta^{*}$-Countability and $\delta^{*}$ Separability). Mathematical Theory and Modeling,.7(1), (2017) 32-37.

[27] Asmhan F. Hassan, Soft Tritopological Spaces. International Journal of Computer Applications, 176(9), (2017) 26-30.

[28] Asmhan F. Hassan, An Introduction to Fuzzy Soft Tritopological Spaces., International Journal of Fuzzy Mathematical Archive, 17(1), (2019) 41-49.

[29] L. A. Al-Swidi and A. S. Saeed, Soft fuzzy and fuzzy soft set theory with applications, Noor Publishing, 2020.

[30] B. Tanay, M. Burc Kandemir, Topological structure of fuzzy soft sets, Computer \& Mathematics with Applications, 61(10), (2011) 2952-2957. 